\title{
Non-Pharmacological Interventions that Improve Quality of Life in People with Mild Cognitive Impairment
}

\author{
En Fu* and Michael Compton \\ Columbia University Vagelos, College of Surgeons and Physicians, USA \\ *Corresponding author: En Fu, Columbia University Vagelos, College of Surgeons and Physicians, USA
}

ARTICLE INFO

Received: 幽 October 19, 2021

Published: October 28, 2021

Citation: En Fu, Michael Compton. Non-Pharmacological Interventions that Improve Quality of Life in People with Mild Cognitive Impairment. Biomed J Sci \& Tech Res 39(4)-2021. BJSTR. MS.ID.006338.

\begin{abstract}
Various non-pharmacological treatment regimens have been developed for people with mild cognitive impairment, but research is lacking regarding whether and how they improve quality of life. This paper reviews published empirical studies and literature reviews. Overall, there is evidence showing that cognitive training, physical exercise, and multi-model programs can improve quality of life in people with mild cognitive impairment. Possible mediators through which those treatments could have effects include depression, sleep quality, and metacognitive factors such as self-efficacy. The need for further research is discussed.
\end{abstract}

Keywords: Mild Cognitive Impairment; Non-Pharmacological Intervention; Older Adults

Abbreviations: MCI: Mild Cognitive Impairment; QOL: Quality of Life; HRQOL: Health-Related Quality of Life; QOL-AD: Quality of Life in Alzheimer's Disease

\section{Introduction}

Mild cognitive impairment (MCI) is the early stage of a number of neurodegenerative diseases characterized by cognitive and functional impairment [1,2]. As lifespan has increased and the aging population grown, MCI has become increasingly prevalent around the world. About $22.3 \%$ to $23.2 \%$ of the US population 65 years of age or older has $\mathrm{MCI}$, with a projected increase of 9.23 million cases from 2020 to 2060 [3]. About 30\% of the older adults recruited from a memory clinic and $5 \%$ of the older adults recruited from the community converted from normal cognition to MCI in a 15-year longitudinal study [4]. Compared to older adults without $\mathrm{MCI}$, those with MCI report lower quality of life (QOL) in physical, psychological, social, and environmental domains [5]. For example, older adults with subjective cognitive decline, MCI, or Alzheimer's disease reported more sleep problems and reduced health-related quality of life (HRQOL) than normal controls [6]. People with
MCI, like those with dementia, have neuropsychiatric symptoms and memory impairment that are persistent despite clinical interventions [7]. Due to this persistence of symptoms, many homebased or community-based interventions have been used to provide patients with the routine care they need. In the current review, we examined empirical studies and review articles that involved nonpharmacological interventions. Although such interventions are often adopted in long-term care of people with MCI [8], a focused review of their effectiveness and mechanism or mediators is still lacking. Moreover, the majority of studies of interventions for patients with MCI have measured cognitive functioning as the primary outcome, and it is not clear to what extent the benefits of community-based interventions can be generalized to other domains of the patient's life [9]. The current review examines whether and how non-pharmacological interventions improve QOL among people with MCI. 


\section{Method}

\section{Eligibility}

Studies published in English-language peer-reviewed journals were included. Eligible studies examined the effect of at least one non-pharmacological intervention on at least one domain of QOL in people with MCI. Clinical trials, quasi-experimental studies, correlational studies, other research reports, and review articles were included. Interventions involving both non-pharmacological interventions and pharmacological or dietary interventions were excluded from the search strategy.

\section{Search Strategy}

PubMed and Google Scholar were used to identify eligible studies published from the earliest available entry through August 2021. The search terms were "mild cognitive impairment," "intervention," and "quality of life." The full text of identified records resulting from the searches were downloaded and stored in the reference manager Zotero. The first author then screened the nonduplicated literature package. Titles and abstracts were screened for eligibility, and the full text of eligible articles were subsequently screened by the first author. The reference lists of eligible articles were screened to identify additional records before screening their full texts.

\section{Information Synthesis}

Synthesis was carried out based on grouping nonpharmacological interventions into main categories of interventions. Then, the mechanisms through which each category of intervention could possibly help improve QOL were examined.

\section{Results}

\section{Study Identification and Screening}

Duplicates and articles only documenting study protocols were removed, resulting in 67 studies for review.

\section{Participants}

The 40 empirical studies included 42,189 participants, who were older adults aged 55 and above, ranging from a mean of 62.1 to 87.9 years.

\section{Study Design}

Twenty-five articles reported randomized, controlled trials or single-case trials. Fifteen articles reported correlational or quasiexperimental studies. Twenty-seven articles were systematic reviews or meta-analyses.

\section{Outcomes}

Among the 40 empirical studies, 36 measured at least one domain of QOL; 12 used the Quality of Life in Alzheimer's Disease (QOL-AD) scale.
Effect of Non-Pharmacological Interventions on the Quality of Life in People with MCI: QOL was rarely a measure of intervention outcomes for people with MCI until about the mid2000s [10]. In 2013, Huckans and colleagues proposed several modifiable protective factors for people with normal cognition to convert to MCI [11], including Mediterranean diet, physical activity, and cognitively stimulating activities. In the past decades, many institutions and health providers have utilized cognitively stimulating activities to improve functioning in people with MCI. Others have attempted to improve functioning by encouraging daily life activities that involve physical exercise. Many nonpharmacological interventions adopted multi-modal approaches to try to improve functioning in people with MCI. In a study by Chandler et al., 272 individuals with MCI participated in a 50hour group-based behavioral intervention program for two weeks. Participants were randomly assigned to one of five groups, with each group lacking one of the five interventions: memory compensation training, computerized cognitive training, support groups, yoga practice, and wellness education [12]. Various functional outcomes of each group were compared against the other groups. The biggest effect size for QOL resulted from the comparison between the "no computerized cognitive training" group and the "no wellness education group," with the former group having better outcomes.

In another study, 32 older adults with MCI received two 8-week comprehensive therapy sessions, each consisted of 30 minutes of physical exercise, a 30-minute reality orientation program, a 30-minute cognitive training program, a 30-minute break, and a 60-minute program that provided reminiscence therapy, cognitive stimulation, and music therapy in turn [13]. Participants in the control group $(n=32)$ received three 3-hour sessions per week for eight weeks consisting of watching general health videos, mild physical exercises, unstructured conversations, and recreational activities. Results showed that those in the experimental group improved significantly more on QOL than those in the control group. A review of 17 RCTs on exercise and cognitive training found that combined exercise and cognitive training improved balance, which is a key risk factor for falls, in older adults with MCI [14]. The most prominent approach to treating MCI is cognitive training. A meta-analysis of 17 studies showed significant benefits of cognitive intervention on MCI. After the cognitive intervention, participants with MCI obtained mild benefits in QOL [15]. In one study, 160 older adults with MCI attended either a 5-week cognitive activity strategy training or a nonspecific education program. The former intervention improved QOL and immediate attention but not general cognitive performance [16]. Such studies suggest that cognitive training that improves various cognitive capacities and cognitive training that improves QOL may be somewhat different and have differing targets. Besides cognitive training, physical exercise is also popular as a recommendation among practitioners and has shown promising treatment effects on QOL 
in people with MCI [17]. Music-making activities had a moderate effect on QOL compared to control conditions [18]. Social dancing is a combination of physical exercise and social activity; research on older Chinese women living in nursing homes showed that participating in Chinese square dancing groups resulted in higher QOL compared to being in a control group that maintained their usual lifestyle [19].

In 2019, a group of 120 older adults in China participated in an RCT in which the intervention group attended a moderatelevel aerobic exercise program and the control group attended a health education program [20]. After 16 weeks, the intervention group showed greater improvements in cognitive function and HRQOL than the control group. The benefits of exercise are even shown in non-intensive physical activities. Eighty older adults with MCI attended a 1-hour Tai Chi session twice weekly for 16 weeks. Compared to participants in the control group, who attended various recreational activities during the same time period, those in the intervention group reported significantly better physical health, mental health, and three domains of HRQOL [21]. Some researchers have suggested that the benefit of exercise on QOL improvement in people with $\mathrm{MCI}$ can be boosted by continuation of physical exercise and have recommended strategies for supporting adherence [22]. However, it is still unknown how effective those adherence support strategies are due to a lack of empirical evidence [23].

How does Non-Pharmacological Intervention Improve QOL in People with MCI?: Multiple mediators have been proposed to explain the treatment effect of non-pharmacological interventions on QOL in people with MCI. A correlational study examining demographic characteristics, physical factors, psychosocial factors, and QOL in older adults with MCI revealed depression and social support as significant predictors of QOL [24]. Thus, it is possible that effective interventions improve QOL in people with MCI by alleviating depressive symptoms and enhancing sense of social support. However, the specific mechanism might be intricate considering evidence showing that interventions designed to alleviate depression and anxiety, but not to enhance social support, failed to improve QOL in people with MCI [25]. The strongest mediator through which non-pharmacological interventions could improve QOL in people with MCI is depression. In the five-group intervention study noted above, the largest effect size resulted from the comparison between the "no computerized cognitive training" group and the "no wellness education" group, with the former reporting higher QOL scores [26]. Consistent with the hypothesis that depressive symptoms might have mediated the relationship between the intervention and QOL improvement, the "no computerized cognitive training" group differed from the "no wellness education" group on measures of mood. Specifically, the group that received wellness education reported higher scores for mood than the group that received computerized cognitive training. Among Korean older adults attending primary care clinics, depression, age, history of stroke, Parkinson's disease, cancer, and diabetes were significant predictors of poorer QOL [5]. An RCT involving 120 Chinese older adults with $\mathrm{MCI}$ found that moderatelevel aerobic exercise improved the participants' HRQOL through reducing depressive symptoms (indirect effect: $\beta=-0.705$ ) and improving sleep quality (indirect effect $\beta=-0.205$ ) [20].

Besides depressive symptoms, several psychological constructs have shown mediation effects between interventions and functioning in people with MCI, including self-efficacy, awareness of deficits, and cognitive techniques to make goals and plans. Correlational studies have shown, in both people with MCI and their peers with normal cognition, that QOL was significantly associated with several psychological constructs and physiological functions [27]. QOL was positively associated with self-efficacy, extraversion personality trait, and agreeableness personality trait, daily life functions, social net, work size, spatial cognition capabilities, and negatively associated with memory complaints, depressive symptoms, and neuroticism personality traits. A survey study showed self-efficacy as a mediator between physical function and perceived QOL in individuals with MCI [28]. However, another correlational study found that anxiety and depression partially mediated the effect of self-efficacy on QOL in people with MCI or dementia, with depression showing a medium mediation effect (b $=0.32,95 \% \mathrm{CI}=[0.23,0.47])$ [29]. Interventions that targeted the general population might be effective in improving QOL in people with MCI because they made the patients less aware of their deficits and symptoms. Research showed that people with MCI or AD who were aware of their diagnosis reported lower QOL in multiple domains than those unaware of their impaired cognition [30]. Moreover, participants who expected their functioning to decline reported greater depression, higher stress, lower QOL, and more cognitive difficulties than participants who were not worrying about their conditions getting worse over time. An empirical study demonstrated the feasibility of a strategy training model for people with MCI to purposefully address barriers in daily activities by developing goals and plans for self-identified impaired processes [31]. Researchers suggested that this model could potentially delay progress from $\mathrm{MCI}$ to dementia via empowering patients to engage in daily activities.

Community-based interventions also carry out QOL-enhancing effects on the individual through the power of neighbors and the sense of being around and engaged with others. In a theoretical discourse, Unger and Wandersman conceptualized the importance of neighbors via their social, cognitive, and affective components [32]. People with a high sense of "neighboring" are more likely to engage in neighborhood support, form social linkages, participate in physical and symbolic communication, and perceive a strong attachment to the community. It is possible that community- 
based interventions could help individuals with MCI improve QOL through neighboring, thus enjoying social support. Another possible pathway through which interventions could improve QOL in people with MCI is by relieving sleep disturbance. Interventions that were effective in reducing sleep disturbance include transcutaneous electrotherapy nerve stimulation, physical exercise, acupressure, mindfulness behavioral therapy, cognitive training, therapies involving light exposure, and therapies that affect multiple functional domains [33]. Therefore, it is possible that physical exercise and cognitive training interventions improve QOL in people with MCI by relieving sleep disturbance. However, existing studies on non-pharmacological interventions of sleep disturbance in people with MCI have had significant clinical and methodological heterogeneity, rendering any comparison among them difficult if not impossible.

\section{Discussion}

Several reviews on interventions among people with $\mathrm{MCI}$ suggest that evidence-based interventions, especially interventions that incorporate standardized tests of non-cognitive measures such as QOL and daily living-related outcomes, should be adopted $[26,34,35]$. Although various cognitive training approaches have been adopted and have shown treatment effects in people with MCI, there is a lack of a theoretical rehabilitation model to guide the development and evaluation of evidence-based cognitive training interventions [11]. There is also a lack of a direct and indirect measures of efficacy or validity of cognitive intervention programs aiming to treat MCI [36]. During program evaluation, factors such as patient gender [37], the matching of intervention type and a measurement instrument [38], and subjective versus objective measures of cognition [8] should be considered. Future research could also explore the possible role of QOL as a mediator in the relationship between social exercise interventions (e.g., Chinese square dancing) and improvement in cognition [19,39].

\section{References}

1. Lombardi G, Crescioli G, Cavedo E, Lucenteforte E, Casazza G, et al. (2020) Structural magnetic resonance imaging for the early diagnosis of dementia due to Alzheimer's disease in people with mild cognitive impairment. Cochrane Database of Systematic Reviews.

2. Orgeta V, McDonald KR, Poliakoff E, Hindle JV, Clare L, et al. (2020) Cognitive training interventions for dementia and mild cognitive impairment in parkinson's disease. Cochrane Database of Systematic Reviews 2: CD011961.

3. Rajan KB, Weuve J, Barnes LL, McAninch EA, Wilson RS, et al. (2021) Population estimate of people with clinical alzheimer's disease and mild cognitive impairment in the united states (2020-2060). Alzheimer's \& Dementia.

4. Chen Y, Denny KG, Harvey D, Farias ST, Mungas D, et al. (2017) Progression from normal cognition to mild cognitive impairment in a diverse clinic-based and community-based elderly cohort. Alzheimer's \& Dementia 13(4): 399-405.
5. Samy AL, Kamaruzzaman SB, Krishnaswamy S, Low W (2020) Predictors of quality of life among older people with mild cognitive impairment attending urban primary care clinics. Clinical Gerontologist 43(4): 441454.

6. Leng M, Yin H, Zhang P, Jia Y, Hu M, et al. (2020) Sleep quality and healthrelated quality of life in older people with subjective cognitive decline, mild cognitive impairment, and Alzheimer disease. Journal of Nervous \& Mental Disease 208(5): 387-396.

7. Ryu S, Ha JH, Park D, Yu J, Livingston G (2011) Persistence of neuropsychiatric symptoms over six months in mild cognitive impairment in community-dwelling Korean elderly. International Psychogeriatrics 23(2): 214-220.

8. Jenkins E, Koirala B, Rodney T, Lee JW, Cotter VT, et al. (2021) Home/ community-based interventions to improve function in persons with mild cognitive impairment/early dementia. Geriatric Nursing 42(5): 1109-1124.

9. Reijnders J, Van Heugten C, Van Boxtel M (2013) Cognitive interventions in healthy older adults and people with mild cognitive impairment: A systematic review. Ageing Research Reviews 12(1): 263-275.

10. Scho“lzel Dorenbos CJM, Van der Steen MJMM, Engels LK, Olde Rikkert MGM (2007) Assessment of quality of life as outcome in dementia and mci intervention trials: A systematic review. Alzheimer Disease \& Associated Disorders 21(2): 172-178.

11. Huckans M, Hutson L, Twamley E, Jak A, Kaye J, et al. (2013) Efficacy of cognitive rehabilitation therapies for mild cognitive impairment (mci) in older adults: Working toward a theoretical model and evidence-based interventions. Neuropsychology Review 23(1): 63-80.

12. Chandler MJ, Locke DE, Crook JE, Fields JA, Ball CT, et al. (2019) Comparative effectiveness of behavioral interventions on quality of life for older adults with mild cognitive impairment: A randomized clinical trial. JAMA Network Open 2(5): 1-12.

13. Han JW, Lee H, Hong JW, Kim K, Kim T, et al. (2016) Multimodal cognitive enhancement therapy for patients with mild cognitive impairment and mild dementia: A multi- center, randomized, controlled, double-blind, crossover trial. Journal of Alzheimer's Disease 55(2): 787-796.

14. Lipardo DS, Aseron AMC, Kwan MM, Tsang WW (2017) Effect of exercise and cognitive training on falls and fall-related factors in older adults with mild cognitive impairment: A systematic review. Archives of Physical Medicine and Rehabilitation 98(10): 2079-2096.

15. Li H, Li J, Li N, Li B, Wang P, et al. (2011) Cognitive intervention for persons with mild cognitive impairment: A meta-analysis. Ageing Research Reviews 10(2): 285-296.

16. Vidovich MR, Lautenschlager NT, Flicker L, Clare L, McCaul K, et al (2015) The pace study: A randomized clinical trial of cognitive activity strategy training for older people with mild cognitive impairment. The American Journal of Geriatric Psychiatry 23(4): 360-372.

17. Demurtas J, Schoene D, Torbahn G, Marengoni A, Grande G, et al. (2020) Physical activity and exercise in mild cognitive impairment and dementia: An umbrella review of intervention and observational studies. Journal of the American Medical Directors Association 21(10): 1415-1422.

18. Dorris JL, Neely S, Terhorst L, VonVille HM, Rodakowski J (2021) Effects of music participation for mild cognitive impairment and dementia: A systematic review and meta-analysis. Journal of the American Geriatrics Society 69(9): 2659-2667.

19. Chang J, Zhu W, Zhang J, Yong L, Yang M, et al. (2021) The effect of Chinese square dance exercise on cognitive function in older women with mild cognitive impairment: The mediating effect of mood status and quality of life. Frontiers in Psychiatry 12: 1-10. 
20. Song D, Yu DSF (2019) Effects of a moderate-intensity aerobic exercise programme on the cognitive function and quality of life of communitydwelling elderly people with mild cognitive impairment: A randomised controlled trial. International Journal of Nursing Studies 93(2019): $97-$ 105.

21. Siu M, Lee DTF (2021) Is Tai Chi an effective intervention for enhancing health-related quality of life in older people with mild cognitive impairment? An interventional study. International Journal of Older People Nursing, 16(5): 1-12.

22. Wang S, Yin H, Meng X, Shang B, Meng Q et al. (2020) Effects of Chinese square dancing on older adults with mild cognitive impairment. Geriatric Nursing 41(3): 290-296.

23. Van der Wardt V, Hancox J, Gondek D, Logan P, Nair R, et al. (2017) Adherence support strategies for exercise interventions in people with mild cognitive impairment and dementia: A systematic review. Preventive Medicine Reports 7: 38-45.

24. Kang Y, Lee E (2018) Quality of life and its factors in korean elderly with mild cognitive impairment. Clinical Nursing Research 27(7): 871-889.

25. Orgeta V, Qazi A, Spector A, Orrell M (2015) Psychological treatments for depression and anxiety in dementia and mild cognitive impairment: Systematic review and meta-analysis. British Journal of Psychiatry 207(4): 293-298.

26. Chandler MJ, Parks AC, Marsiske M, Rotblatt LJ, Smith GE (2016) Everyday impact of cognitive interventions in mild cognitive impairment: A systematic review and meta-analysis. Neuropsychology Review 26(3): 225-251.

27. Maki Y, Yamaguchi T, Yamagami T, Murai T, Hachisuka K, et al. (2014) The impact of subjective memory complaints on quality of life in communitydwelling older adults: Memory complaints and QOL in the elderly. Psychogeriatrics 14(3): 175-181.

28. Langer K, Oshea DM, De Wit L, De Feis B, Mejia A, et al. (2019) Selfefficacy mediates the association between physical function and perceived quality of life in individuals with mild cognitive impairment. Journal of Alzheimer's Disease 68(4): 1511-1519.

29. Tonga JB, Eilertsen D, Solem IKL, Arnevik EA, Korsnes MS, et al. (2020) Effect of self-efficacy on quality of life in people with mild cognitive impairment and mild dementia: The mediating roles of depression and anxiety. American Journal of Alzheimer's Disease \& Other Dementias ${ }^{\circledR}$ 35: $1-10$.

\section{ISSN: 2574-1241}

DOI: 10.26717/BJSTR.2021.39.006338

En Fu. Biomed J Sci \& Tech Res

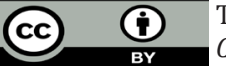

This work is licensed under Creative

Commons Attribution 4.0 License

Submission Link: https://biomedres.us/submit-manuscript.php
30. Stites SD, Karlawish J, Harkins K, Rubright JD, Wolk D (2017) Awareness of mild cognitive impairment and mild alzheimer's disease dementia diagnoses associated with lower self-ratings of quality of life in older adults. The Journals of Gerontology: Series B 72(6): 974-985.

31. Rodakowski J, Reynolds CF, Lopez OL, Butters MA, Dew MA, et al. (2018) Developing a non-pharmacological intervention for individuals with mild cognitive impairment. Journal of Applied Gerontology 37(5): 665676.

32. Unger DG, Wandersman A (1985) The importance of neighbors: The social, cognitive, and affective components of neighboring. American Journal of Community Psychology 13(2): 139-169.

33. Ocaoimh R, Mannion H, Sezgin D, Odonovan MR, Liew A, et al. (2019) Non-pharmacological treatments for sleep disturbance in mild cognitive impairment and dementia: A systematic review and meta-analysis. Maturitas 127(2019): 82-94.

34. Couch E, Lawrence V, Co M, Prina M (2020) Outcomes tested in nonpharmacological interventions in mild cognitive impairment and mild dementia: A scoping review. BMJ Open 10(4): e035980.

35. Harrison JK, Noel Storr AH, Demeyere N, Reynish EL, Quinn TJ (2016) Outcomes measures in a decade of dementia and mild cognitive impairment trials. Alzheimer's Research \& Therapy 8(1): 48.

36. Jean L, Bergeron M, Thivierge S, Simard M (2010) Cognitive intervention programs for individuals with mild cognitive impairment: Systematic review of the literature. The American Journal of Geriatric Psychiatry 18(4): 281-296.

37. Pusswald G, Tropper E, Kryspin Exner I, Moser D, Klug S, et al. (2015) Health-related quality of life in patients with subjective cognitive decline and mild cognitive impairment and its relation to activities of daily living. Journal of Alzheimer's Disease 47(2): 479-486.

38. Pusswald G, Moser D, Pflüger M, Gleiss A, Auff E, et al. (2016) The impact of depressive symptoms on health-related quality of life in patients with subjective cognitive decline, mild cognitive impairment, and Alzheimer's disease. International Psychogeriatrics 28(12): 2045-2054.

39. Poon P, Hui E, Dai D, Kwok T, Woo J (2005) Cognitive intervention for community-dwelling older persons with memory problems: Telemedicine versus face-to-face treatment. International Journal of Geriatric Psychiatry 20(3): 285-286.

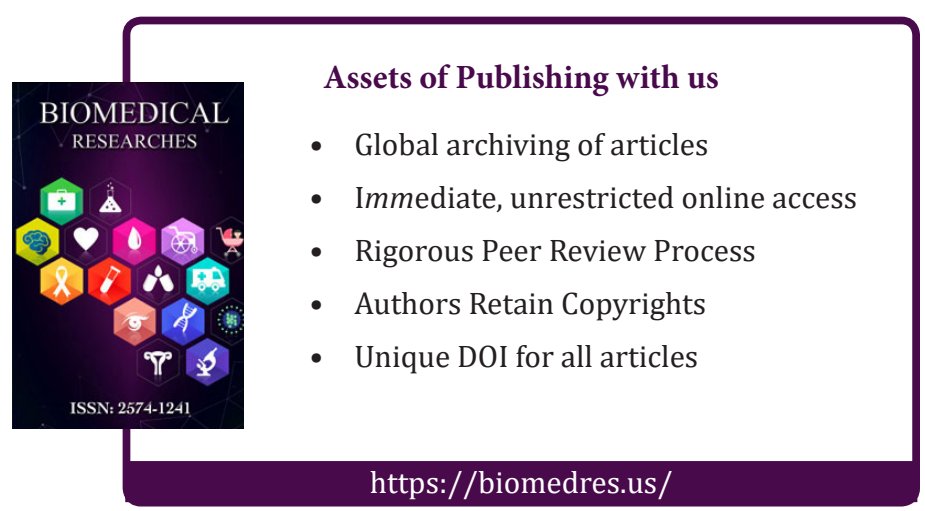

Copyright@ En Fu | Biomed J Sci \& Tech Res | BJSTR. MS.ID.006338. 